\title{
The Co-Occurrence of Sexsomnia, Sleep Bruxism and Other Sleep Disorders
}

\author{
Helena Martynowicz ${ }^{1}$, Joanna Smardz ${ }^{2}$, Tomasz Wieczorek ${ }^{3}$, Grzegorz Mazur ${ }^{1}$, Rafal Poreba ${ }^{1}$, \\ Robert Skomro ${ }^{4}$, Marek Zietek ${ }^{5}$, Anna Wojakowska ${ }^{1}$, Monika Michalek ${ }^{1}$ (i) and \\ Mieszko Wieckiewicz ${ }^{2, *}$ \\ 1 Department of Internal Medicine, Occupational Diseases and Hypertension, Wroclaw Medical University, \\ 50-367 Wroclaw, Poland; helenamar@poczta.onet.pl (H.M.); grzegorzmaz@yahoo.com (G.M.); \\ sogood@poczta.onet.pl (R.P.); ania.wojakowska@wp.pl (A.W.); monika.michalek1@gmail.com (M.M.) \\ 2 Department of Experimental Dentistry, Wroclaw Medical University, 50-367 Wroclaw, Poland; \\ joannasmardz1@gmail.com \\ 3 Department and Clinic of Psychiatry, Wroclaw Medical University, 50-367 Wroclaw, Poland; \\ dobrewieczorki@gmail.com \\ 4 Division of Respiratory Critical Care and Sleep Medicine, Department of Medicine, \\ University of Saskatchewan, Saskatoon, SK S7N 5A2, Canada; r.skomro@usask.ca \\ 5 Department of Periodontology, Wroclaw Medical University, 50-367 Wroclaw, Poland; \\ marekzietek@gazeta.pl \\ * Correspondence: m.wieckiewicz@onet.pl; Tel.: +48-660-47-87-59
}

Received: 3 August 2018; Accepted: 20 August 2018; Published: 23 August 2018

\begin{abstract}
Background: Sleep sex also known as sexsomnia or somnambulistic sexual behavior is proposed to be classified as NREM (non-rapid eye movement) parasomnia (as a clinical subtype of disorders of arousal from NREM sleep-primarily confusional arousals or less commonly sleepwalking), but it has also been described in relation to REM (rapid eye movement) parasomnias. Methods: The authors searched the PubMed database to identify relevant publications and present the co-occurrence of sexsomnia and other sleep disorders as a non-systematic review with case series. Results: In the available literature the comorbidity of sexsomnia and other sleep disorders were reported mainly in case reports and less in case series. Sexsomnia was reported both with one and with multiple sleep-related disorders, with NREM parasomnias and obstructive sleep apnea (OSA) being the most commonly reported. Furthermore, the authors enrich the article with new findings concerning two novel cases of sleep bruxism triggering recurrent sexsomnia episodes. Conclusions: Sexsomnia has still not been reported in the literature as often as other parasomnias. The coexistence of sexsomnia and other sleep-related disorders should be more thoroughly examined. This could help both in sexsomnia as well as other sleep-related disorders management.
\end{abstract}

Keywords: sexsomnia; sleep sex; sleep bruxism; sleepwalking; sleep talking; obstructive sleep apnea; video-polisomnography

\section{Introduction}

Parasomnias comprise a category of sleep disorders with abnormal physical events (movements or behaviors), experiences (dreams, emotions, perceptions), and autonomic nervous system activity. They can appear during entry into sleep, within sleep or during arousals [1,2]. Most parasomnias are dissociated sleep states which are partial arousals during the transitions between wakefulness and non-rapid eye movement (NREM) sleep, or wakefulness and rapid eye movements (REM) sleep [1]. That is why parasomnias can be divided into NREM sleep, rapid eye movement (REM) sleep, and other parasomnias (which usually appear in different sleep stages or during sleep onset or offset) [1]. There 
are two classifications of sleep disorders: Diagnostic and Statistical Manual of Mental Disorders, fifth edition (DSM-5) classification [3] and the International Classification of Sleep Disorders, 3rd edition (ICSD-3) [2].

Sleep sex is also known as sexsomnia, or somnambulistic sexual behavior is proposed to be classified as a NREM parasomnia (as a clinical subtype of disorders of arousal from NREM sleep—primarily confusional arousals or less commonly sleepwalking), but it has also been described in relation to REM parasomnias [4-10].

Sexsomnia has still not been reported in the literature as often as other parasomnias. The population prevalence is unknown. It can be based only on the estimated overall prevalence of parasomnias, which for adults is estimated to range between $2 \%$ and $6 \%$ [6]. The aim of this study was to investigate the co-occurrence of sexsomnia and other selected sleep disorders and present it as a non-systematic review with case series, including two novel cases from our center of sleep bruxism triggering recurrent sexsomnia episodes, as documented by video-polysomnography.

\section{Methods}

The authors used the following inclusion criteria: studies that discussed the co-occurrence of sexsomnia and other sleep disorders and behaviours. English language and full-text articles published between January 2003 and June 2018 were included in this non-systematic review. Participants were males and females of any age with a clinical diagnosis of sexsomnia. The authors searched the PubMed database to identify relevant publications. The literature search strategy was conducted as follows: each of two synonymous phrases, i.e., (1) sexsomnia, (2) sleep sex, were combined with each of: (a) sleep disorder, (b) sleep disorders, e.g., "sexsomnia sleep disorder", viz. (1) + (a); "sexsomnia sleep disorders", viz. (1) + (b), etc. Four queries were obtained. The authors screened the reference list of included studies to identify other potentially useful studies. Firstly, the authors screened the titles and abstracts, then, the full texts for keywords, such as "sexsomnia", "sleep sex" to find those that were potentially suitable. The data extraction process focused on the information about each study type and coexisting sleep disorder. The authors also described two clinical cases of patients in whom sexsomnia co-occurred with bruxism and other sleep-related disorders.

\section{Results}

In 1875, Moet reported abnormal sexual activity during sleep for the first time in history [5]. In the available literature, the comorbidity of sexsomnia and other sleep disorders is reported mainly in case reports and less in case series. In some studies, the authors focused more on treatment than the coexistence of sexsomnia with other sleep disorders. Ariño et al. described four cases of sexsomnia. In one patient clonazepam treatment was started and reported to decrease the frequency of both confusional arousals and sexsomnia episodes [11]. Also, Béjot et al. described two cases of sexsomnia in adult women that were successfully treated with selective serotonin reuptake inhibitors (SSRI) [12]. However, a case of SSRI-induced sexsomnia has been also reported [13].

\subsection{Sexsomnia and Obstructive Sleep Apnea/Hypopnea}

Obstructive sleep apnea (OSA) is the most common type of sleep apnea and is caused by complete or partial obstructions of the upper airway. It is characterized by repetitive episodes of shallow or paused breathing during sleep, despite the effort to breathe, and is usually associated with a reduction in blood oxygen saturation [2]. The co-occurrence of sexsomnia and obstructive sleep apnea (OSA) was the second most commonly reported, after NREM parasomnias. Meira et al. presented a case of a patient with mild OSA and sexsomnia who had the resolution of both conditions with a mandibular advancement device (MAD) [14]. Another case of successful MAD treatment was described by Khawaja et al. A patient with OSA and sexsomnia with no signs of disordered arousals was treated with MAD, but after five months requested continuous positive airway pressure (CPAP) treatment due to mandibular pain. CPAP treatment turned out to be successful as well [15]. Another case of successful 
treatment of moderate OSAS and sexsomnia (4-year history before treatment-started together with snoring) was with nasal CPAP as described by Schenck [16]. Successful treatment with nasal CPAP of both disorders was also reported by Della Marca [17]. Two additional cases of such coexistence were described by Ariño, though both patients refused treatment for OSA and sexsomnia [11].

\subsection{Sexsomnia and Sleep-Related Eating Disorder}

Sleep-related eating disorder (SRED) is a condition characterized by recurrent episodes of eating at the transition from night-time sleep to arousal. SRED patients describe eating in an out-of-control manner with a preference for high-caloric foods and sometimes with inedible or toxic items $[2,18]$. Gomis et al. reported a case of a 41-year-old woman with a diagnosis of narcolepsy with mild cataplexy. After introducing treatment with sodium oxybate, the patient reported sleep-related sexual episodes after which the sleep-related eating episode took place [10].

\subsection{Sexsomnia and Sleepwalking}

Sleepwalking is also known as noctambulism or somnambulism. It is a phenomenon of combined sleep and wakefulness. It is classified as parasomnia sleep disorder. Yeh et al. reported a case of a 20-year-old soldier presenting sexsomnia and sleepwalking. Researchers tried clonazepam treatment. The treatment controlled the sleepwalking, but not the sleep masturbation episodes [19].

\subsection{Sexsomnia and Multiple Various Sleep-Related Disorders}

In some cases, sexsomnia occurs with not one, but several other sleep-related disorders. Soca et al. presented a case of a 42-year-old male patient with sexsomnia as a part of parasomnia overlap disorder (POD). The patient had sleepwalking, sleep-related eating, confusional arousals, sleep talking, and REM sleep behavior disorder (RBD). In this patient OSA was also diagnosed, which played a promoting role in the sleepwalking and sleep-related eating, as there was a good response to nasal continuous positive airway pressure (nCPAP). Although this therapy did not give an effect on the sexsomnia treatment, it did respond to the bedtime clonazepam therapy [20]. Also, Pelin et al. reported a case of a 31-year-old male who presented sexsomnia with sleepwalking and sleep talking. Sexsomnia, in this case, was treated with carbamazepine [21]. One case presented with a complex history of POD, including sleepwalking, sexsomnia and REM sleep behavior disorder, coexisting with mild OSA. Parasomnias did not respond to nasal CPAP treatment, but there was an improvement after clonazepam administration [22]. Shapiro et al. reported a case of the coexistence of sexsomnia, OSA and sleepwalking, with good response for CPAP. What is more intriguing, the patient ceased therapy because of discomfort-at this moment sexsomnia relapsed, until the treatment reinstitution [23]. Irfan et al. described a case of sleep-related orgasms in a 57-year-old woman who was also diagnosed with hypnic jerks, exploding head syndrome and OSA. In this case, bedtime Clonazepam therapy was effective in sleep-related orgasms and hypnic jerks treatment [24]. Clonazepam therapy was not effective in another case presented by Mioč et al. they described a 33-year-old man presenting episodes of sexsomnia, hypnagogic hallucinations, sleep paralysis, night terrors and sleepwalking. In this patient, further studies led to the diagnosis of sleep-related hypermotor epilepsy. Starting antiepileptic treatment had to be discontinued because of increased violence of the episodes [25].

Data extraction of selected studies is presented in Table 1. 
Table 1. Data extraction of selected studies.

\begin{tabular}{ll}
\hline \multicolumn{1}{c}{ Study, Year } & \multicolumn{1}{c}{ Sleep Disorders That Co-occurred with Sexsomnia } \\
\hline Meira et al., 2016 [14] & Obstructive sleep apnea (OSA) \\
Khawaja et al., 2017 [15] & Obstructive sleep apnea (OSA) \\
Schenck et al., 2008 [16] & Obstructive sleep apnea (OSA) \\
Della Marca et al., 2009 [17] & Obstructive sleep apnea (OSA) \\
Ariño et al., 2014 [11] & Obstructive sleep apnea (OSA) \\
Gomis et al., 2016 [10] & Sleep-related eating disorder (SRED) \\
Yeh et al., 2016 [19] & Sleepwalking \\
Soca et al., 2016 [20] & Sleepwalking, sleep-related eating disorder, confusional arousals, sleep talking, REM \\
Pelin et al., 2012 [21] & Sleep behavior disorder (RBD), obstructive sleep apnea (OSA) \\
Cicolin et al., 2011 [22] & Sleepwalking and sleep talking \\
Shapiro et al., 2003 [23] & Sleepwalking, obstructive sleep apnea (OSA) \\
Irfan et al., 2018 [24] & Hypnic jerks, exploding head syndrome, obstructive sleep apnea (OSA) \\
Mioč et al., 2018 [25] & Hypnagogic hallucinations, sleep paralysis, night terrors, sleepwalking, sleep-related \\
& hyperomotor epilepsy \\
\hline
\end{tabular}

\subsection{Co-Occurrence of Sexsomnia, Sleep Bruxism and Other Sleep-Related Disorders}

The authors will now present two novel case reports that are part of the research project titled "Evaluation of the Quality of Sleep, Endothelial Function, Cardiovascular Risk, Thyroid Function, a Function of Masticatory Muscles and Psycho-emotional State of Patients with Sleep Bruxism". The project was performed in the Department and Clinic of Internal and Occupational Diseases and Hypertension, Wroclaw Medical University, Wroclaw, Poland. The project was co-financed from funds for young researchers obtained from the Wroclaw Medical University (STM.B022.17.011). The project was accepted by the local Ethical Committee (ID KB-195/2017) and registered on the ClinicalTrials.gov (ID WMU1/2017).

Both of the presented cases show the co-occurrence of sexsomnia and sleep-related bruxism. According to consensus established in 2017 bruxism is a common phenomenon and can be defined as a repetitive jaw-muscle activity characterized by clenching or grinding of the teeth and/or by bracing or thrusting of the mandible. This activity can occur both during sleep time (sleep bruxism (SB)) and during wakefulness (awake bruxism (AB)). Its prevalence ranges from 8 to $31 \%$ in the adult population with no gender differences. There are many factors involved in bruxism's etiology. They can be divided into biological (i.e., neurotransmitters, sleep arousals), psychological (i.e., stress, anxiety, personality) and exogenous factors (i.e., nicotine, alcohol, drugs, medications) [26-29]. According to the newest commentary to the international consensus on the assessment of bruxism sleep and awake bruxism are generally considered as different behaviors observed during sleep and wakefulness, and that is why the earlier common definition should be separated into two. The new recommended definition of sleep-related bruxism is: "Masticatory muscle activity during sleep that is characterized as rhythmic (phasic) or non-rhythmic (tonic) and is not a movement disorder or a sleep disorder in otherwise healthy individuals" [30].

\subsubsection{Case 1}

The patient was a 49-year-old Caucasian male, a professional driver, who underwent a dental examination in which bruxism was detected [29-31]. He underwent several laboratory tests (in order to assess metabolic and hormonal functions), whole-night video-polysomnography (vPSG) adjusted to evaluating bruxism and parasomnias. PSG was evaluated in $30 \mathrm{~s}$ epochs, according to standard sleep criteria. Pathological events were evaluated according to the standards of the American Academy of Sleep [32]. The patient was also examined with a battery of scales and questionnaires: Athens Insomnia Scale (AIS), Epworth Sleepiness Scale (ESS), Berlin Questionnaire, STOP BANG questionnaire, Beck Depression Inventory (BDI), WHO Quality of Life-BREF (WHO QOL-BREF), Pittsburgh Sleep Quality Index (PSQI), Headache Impact Test-6 (HIT-6), Oral Behavior Checklist, Paris Arousal Disorder Severity Scale (PADSS) and Perceived Stress Scale-10 (PSS-10). 
Medical history was positive for hypertension, psoriasis and Barrett's esophagus. The patient reported a history of common nightmares and sleep terrors. He was also a long-term smoker. Medications included pantoprazole $(40 \mathrm{mg})$, nebivolol $(5 \mathrm{mg})$ and ramipril $(5 \mathrm{mg})$. At presentation he complained of snoring, daytime fatigue, clenching and grinding of the teeth (during the day as well as night), pain located bilaterally in masseter muscles and area of temporomandibular joints, perceived increased masseter muscle tension and dissatisfaction with his quality of sleep. He identified his bruxism as one of the main causes of his subjectively reduced sleep efficiency.

Dental examination confirmed malocclusion (Angle's class II), dental crowding, excessive overbite, teeth midline deviation and high Tooth Wear Index. During the examination, according to the Diagnostic Criteria for Temporomandibular Disorders (DC/TMD) guidelines, we found pain in both masseters and pain of both temporomandibular joints and also clicks in both temporomandibular joints during opening and closing of the mouth and during lateral and protrusive movements [31]. The Oral Behavior Checklist showed that the patient presented behaviors strictly connected with bruxism, such as bracing or thrusting of the mandible during the daytime. The patient knew about his bruxism and noticed an increased number and frequency of episodes after changing to a more stressful job. Dental examination together with Oral Behavior Checklist confirmed the high probability of severe sleep and awake bruxism.

Physical exam was within normal limits (WNL), apart from increased BMI (29.3). ESS score was 11 (mild daytime sleepiness). STOP BANG (patient scored 5 points) and Berlin questionnaires confirmed increased risk of Obstructive Sleep Apnea Syndrome (OSAS). PSS-10 indicated a higher level of perceived stress (21 points); BDI score of 9 was in the non-depressed range. Laboratory tests revealed only lowered concentration of HDL cholesterol, slight hypertriglyceridemia and slightly increased fasting glucose levels with normal levels of hormones and other metabolic parameters.

V-PSG confirmed the diagnosis of severe SB (Bruxism Episodes Index $=10.1 / \mathrm{h}$, Bruxism Bursts Index $=12.8 / \mathrm{h}$ ), with total count of 64 episodes lasting from $1.8 \mathrm{~s}$ up to $21.5 \mathrm{~s}$ (mean $=6.5 \mathrm{~s}$ ). They often triggered EEG arousals. Suspected OSAS was not confirmed (AHI $=4.8 / \mathrm{h}$, average $\mathrm{SpO}_{2}=92.5 \%$, Oxygen Desaturation Index $=5.5 / \mathrm{h}$, average desaturation drop $=2.9 \%$ ). Total Sleep Time (TST) was $379 \mathrm{~min}$, Sleep Latency (SL) was $31 \mathrm{~min}$ and REM Latency (RL) was $160 \mathrm{~min}$. Sleep stage distributions: $\mathrm{N} 1=6.9 \%$; $2=60.1 \% ; \mathrm{N} 3=10 \%$; REM sleep $=23 \%$. Sleep moisturance is presented in Figure 1 . Periodic limb movements (PLMs) were not evaluated due to technical limitations. PSG report is presented in Table 2.

Table 2. Polysomnography report.

\begin{tabular}{cc}
\hline Index & Value \\
\hline Sleep latency & $30.7 \mathrm{~min}$ \\
REM latency & $159.5 \mathrm{~min}$ \\
AHI & $4.8 / \mathrm{h}$ \\
ODI & $5.5 / \mathrm{h}$ \\
Average desaturation drop & $2.9 \%$ \\
Average $\mathrm{SpO}_{2}$ & $92.5 \%$ \\
Minimum $\mathrm{SpO}_{2}$ & $88 \%$ \\
Bruxism Episodes Index & $10.1 / \mathrm{h}$ \\
Bruxism Burst Index & $12.8 / \mathrm{h}$ \\
\hline
\end{tabular}

REM, Rapid Eye Movement; AHI, Apnea/Hypopnea Index; ODI, Oxygen Desaturation Index; $\mathrm{SpO}_{2}$, Saturation. 


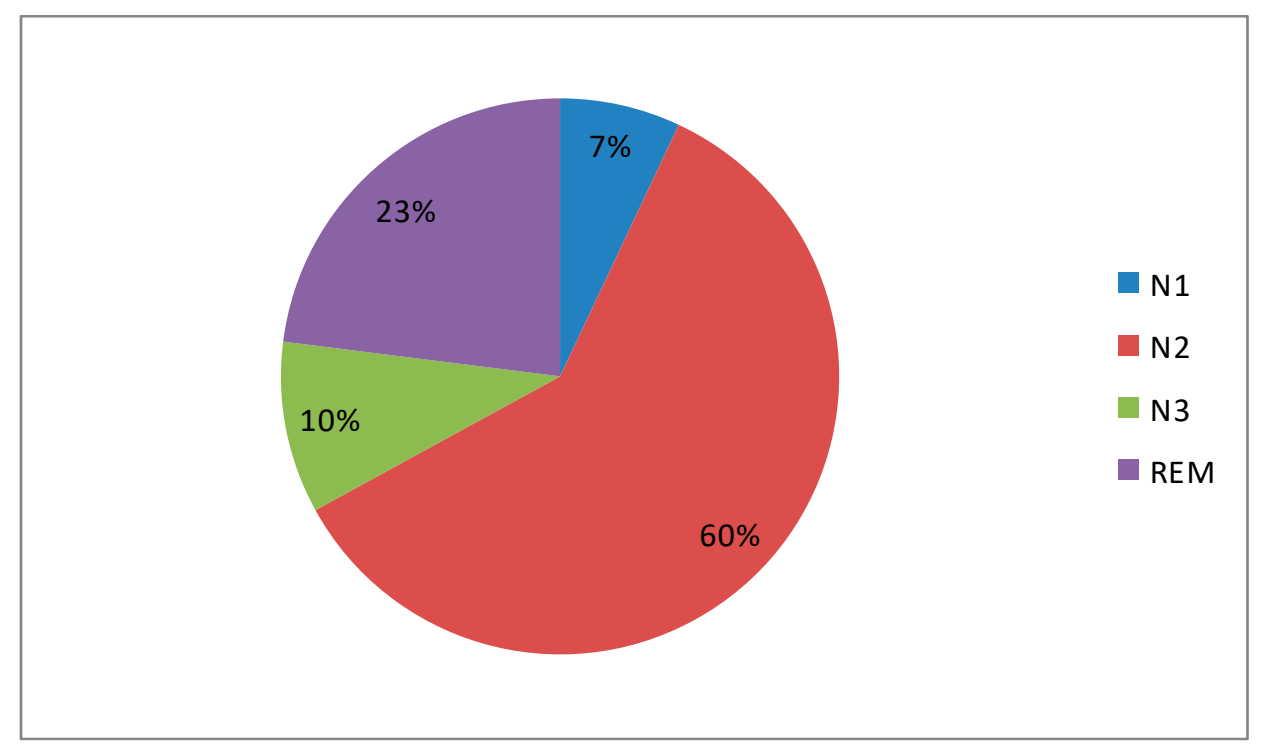

Figure 1. Sleep microstructure. N1, non-rapid eye movement stage 1; N2, non-rapid eye movement stage 2 ; N3, non-rapid eye movement stage 3; REM, rapid eye movement stage.

The most unexpected and striking result was observed with the video recording. The patient had several episodes (total count $=8$ ) of sexual activity, lasting from a few up to over a dozen seconds, which appeared during most sleep stages (N1, N2, and REM). The patient performed masturbation with his hand or with friction moves by pushing his loins against the quilt while lying in the prone position or on his side. The episodes were short, mostly lasting less than $20 \mathrm{~s}$, and did not result in ejaculation. Notably, each of these sleep masturbation episodes was preceded by a bruxism episode with EEG arousal lasting for at least a few seconds. In most of the episodes, SB lasted throughout the whole sexsomnia episode. After awakening from all of these episodes, there was never any recall by the patient. There were no spontaneous arousals from N3 or N2 sleep, and REM-atonia was preserved. However, an episode of intermittent non-periodic myoclonic activity was recorded in N2, lasting $21 \mathrm{~min}$ and about 40 myoclonic movements in the left arm and hand were recorded. This episode ended with SB and an awakening. Mentioned episodes are presented in the Supplementary Figures S1-S3.

The patient was offered various treatments, including manual therapy jaw massages and an occlusal splint for the lower teeth arch with the goal of relaxing the masticatory muscles and decrease the number of SB/AW episodes, but he refused. When informed about the SB-triggered sexsomnia episodes, he confirmed that he was unaware and added that he would always ask for a single room in hotels.

\subsubsection{Case 2}

The patient was a 38-year-old Caucasian male, who underwent a thorough dental examination in which bruxism was detected [29-31]. He underwent several laboratory tests (in order to assess metabolic and hormonal functions), whole-night vPSG adjusted to evaluating bruxism, OSAS and parasomnias. PSG was evaluated in $30 \mathrm{~s}$ epochs, according to standard sleep criteria. Pathological events were evaluated according to the standards of the American Academy of Sleep [32]. The patient was also examined with the same battery of scales and questionnaires that were mentioned in Case 1. Medical history was positive for masseter muscles hypertrophy and severe teeth damage. The patient reported a history of common nightmares and muscle cramps during sleep. He was also a long-term smoker. At presentation, he complained of loud snoring, daytime fatigue and massive teeth wear.

Dental examination confirmed bilateral hypertrophy of masseter muscles and teeth wear of 4th stage in Tooth Wear Index (Figure 2). 


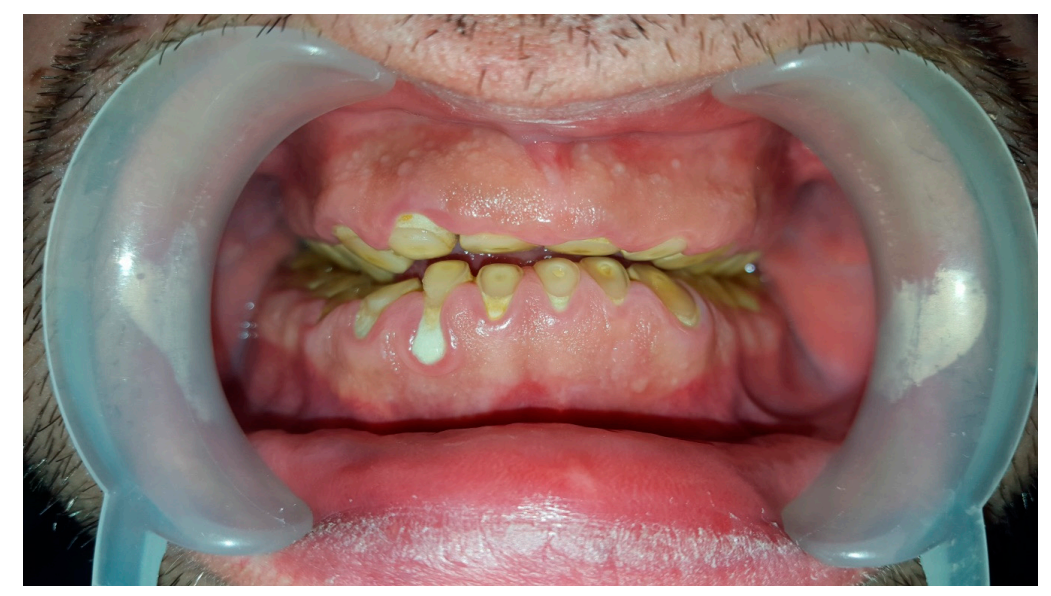

Figure 2. Tooth wear in presented bruxist patient.

Physical examination revealed enlarged palatine tonsils, apart from this examination was WNL, with Body-Mass Index 25.3. The ESS score was 16 and confirmed severe, excessive daytime sleepiness. STOP BANG confirmed high risk of OSAS, same with Berlin Questionnaire (2 categories positive). PSS-10 indicated a level of perceived stress within normal limits (19 points); BDI score of 7 was in the non-depressed range, while the PSQI score of 6 points confirmed a slightly decreased sleep quality. Laboratory tests revealed only lowered concentration of 25-hydroxycholecalciferol.

V-PSG confirmed the diagnosis of severe SB (Bruxism Episodes Index $=11.4$ episodes $/ \mathrm{h}$, Bruxism Bursts Index $=3.1 / \mathrm{h}$ ), with a total count of 69 episodes lasting from $2.4 \mathrm{~s}$ and up to $19.8 \mathrm{~s}$ (mean $=8 \mathrm{~s}$ ). Most of the registered bruxism episodes were associated with respiratory events and spontaneous arousals. Also, the suspected OSAS was confirmed and evaluated as severe with the $\mathrm{AHI}=33.5 / \mathrm{h}$, average $\mathrm{SpO}_{2}=92.2 \%$, Oxygen Desaturation Index $=35.7 / \mathrm{h}$, average desaturation drop $=6.1 \%$ and percentage of sleep time with blood oxygen saturation below $90 \%=13.4 \%$. Apnea to Bruxism Index $=7.6 / \mathrm{h}$. Desaturations often triggered spontaneous arousals, with a total count of 57 arousals on the Arousal Index $=9.4$. Total Sleep Time (TST) was $363.5 \mathrm{~min}$, Sleep Latency (SL) was $10.5 \mathrm{~min}$, and REM Latency (RL) was $59.5 \mathrm{~min}$. Sleep stage distributions: $\mathrm{N} 1=9.4 \%$; N2 $=50.5 \% ; \mathrm{N} 3=18 \%$; and REM sleep $=22.1 \%$ (Figure 3). Pulse rate reached values between 60 and 103, with average $=75.6$. Periodic limb movements (PLMs) were not evaluated due to technical limitations. PSG report is presented in Table 3.

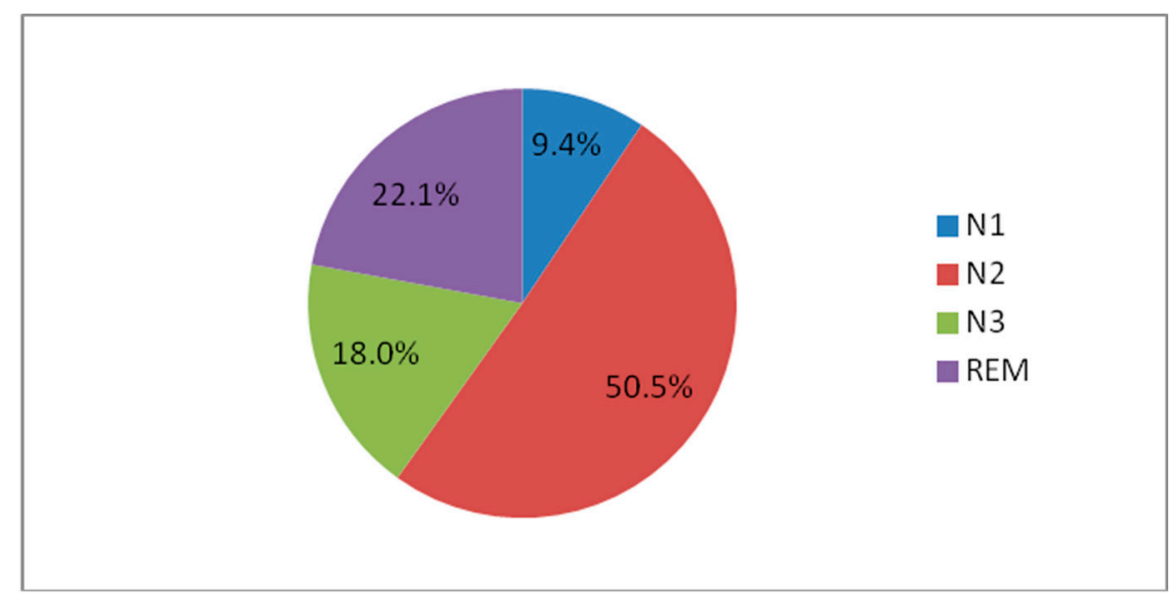

Figure 3. Sleep microstructure. N1, non-rapid eye movement stage 1; N2, non-rapid eye movement stage 2; N3, non-rapid eye movement stage 3; REM, rapid eye movement stage. 
Table 3. Polysomnography report.

\begin{tabular}{cc}
\hline Index & Value \\
\hline Sleep latency & $10.5 \mathrm{~min}$ \\
REM latency & $59.5 \mathrm{~min}$ \\
AHI & $33.5 / \mathrm{h}$ \\
ODI & $35.7 / \mathrm{h}$ \\
Average desaturation drop & $6.1 \%$ \\
Average SpO 2 & $92.2 \%$ \\
Percentage of sleep time with saturation below $90 \%$ & $13.4 \%$ \\
Bruxism Episodes Index & $11.4 / \mathrm{h}$ \\
Bruxism Burst Index & $3.1 / \mathrm{h}$
\end{tabular}

REM, Rapid Eye Movement; AHI, Apnea/Hypopnea Index; ODI, Oxygen Desaturation Index; $\mathrm{SpO}_{2}$, Saturation.

In the video recording, we observed repeated episode of OSAS, SB and sexsomnia overlapping. There was a single period lasting about 17 min divided into several repeatable and similar episodes. The first episode started with hypopnea during the N2 stage in a supine position. When the patient reached a blood oxygen saturation level of $88 \%$, spontaneous arousal occurred lasting $5 \mathrm{~s}$ and was accompanied by a mixed bruxism episode lasting $15 \mathrm{~s}$. After $3 \mathrm{~s}$ of bruxism activity, the patient started to move his left arm and then masturbated with his dominant left hand. The whole motor activity of the arm lasted about $20 \mathrm{~s}$ and ended suddenly, right before the beginning of apnea episode. PSG recording with EEG of this event is presented in the Supplementary Figure S4. It started with blood oxygen saturation of $95 \%$ and lasted $35 \mathrm{~s}$, during which the patient desaturated down to $86 \%$ and the apnea ended with the patient's sudden gasp, accompanied by tonic bruxism episode (10 s) and again sexual motor activity lasting few seconds. There was a total period of $22 \mathrm{~s}$ of breathing and snoring, and then the next apnea appeared, lasting $33 \mathrm{~s}$. Respiratory registration on the first two events are presented in the Supplementary Figure S5. We observed next 14 episodes which looked similar-first apnea/hypopnea appeared, during which saturation went down to about $89 \%$ and then breathing occurred with a desaturation drop, still decreasing down to $85-87 \%$. During this desaturation drop bruxism and sexual activity appeared, but lasted only until the patient reached a saturation of $89-90 \%$ again. Then the activity stopped with the continuation of breathing for a few seconds until the next episode of apnea appeared. What is striking about this vPSG recording is the recurrence of these very similar episodes, each event happening within the same scheme, although during the last set of episodes normal breathing decreased between apneas and became shorter and shorter, from $28 \mathrm{~s}$ down to 9-10 s. After the last episode, the patient changed his position to non-supine and breathed normally, with no bruxism or sexsomnia until the next change of position. However, during the rest of the sleep time, no sexual activity was observed. Sexual activity was not leading to ejaculation, and it mostly appeared during the N2 stage or N1 stage, which was present after some spontaneous arousals evoked by respiratory events, similar to SB episodes. For the most of the time of sexual activity also the bruxism activity was present. The patient did not remember the episode. Respiratory registration of the whole described episode is presented in the Supplementary Figure S6.

The patient was offered treatment with continuous positive airway pressure (CPAP) according to the recommendations of the American Academy of Sleep Medicine. The first night with treatment resulted in a good response to treatment. Tonsillectomy was also advised. Dental treatment could be performed after OSA treatment and sleep bruxism management.

Both of the cases describe the coexistence of bruxism and sexsomnia. The mechanism of comorbidity of these both sleep disorders cannot be accurately determined because there are no other similar published clinical cases and what is more important, the mechanisms for generating sexsomnia instead of other parasomnia behaviors is still unknown. We suspect that the SB arousal can trigger sexual behaviors. However, these two cases (adult males, 39 and 49 years old) present the typical profile of sexsomnia that is more often connected with the male gender (approx. $75 \%$ in 
published cases), while there are notably no gender differences in $\mathrm{SB}$, so that could have created a bias for sexsomnia episodes induced by SB arousals. Accordingly the presented cases, SB can be added to comorbid with sexsomnia sleep disorders, and the problem of comorbidity of these two issues should be studied more precisely.

\section{Conclusions}

Sexsomnia has still not been reported in the literature as often as other parasomnias. In most studies, it is reported as a case report or case series which is the main limitation. The coexistence of sexsomnia and other sleep-related disorders should be more thoroughly examined. This could help, both in sexsomnia, as well as in the treatment of other sleep-related disorders. Patients with established sexsomnia should undergo video-polysomnography to identify the possible coexistence of other sleep disorders, and its cause and effect reference to sexsomnia episodes. It would also be advised to perform screening of sexsomnia in patients with other sleep-related disorders, to establish a larger group of cases with such coexistence for further analysis and the improvement of treatment. The implementation of questions about the presence of possible abnormal sexual behavior during sleep and asking the patient's partner may also be helpful.

Supplementary Materials: The following are available online at http:/ /www.mdpi.com/2077-0383/7/9/233/s1, Figure S1: Case 1. Sexsomnia episode on N1/N2 transition with bruxism activity preceding the episode, Figure S2: Case 1. Bruxism episode followed by sexsomnia in REM sleep, Figure S3: Case 1. Myoclonic activity-21 min episode, Figure S4: Case 2. EEG of a single sexsomnia event, Figure S5: Case 2. First two sexsomnia events, Figure S6: Case 2. Whole sexsomnia episode.

Author Contributions: Conceptualization, G.M., M.W., H.M.; Methodology, J.S., T.W.; Validation, M.Z., R.S. and R.P.; Formal Analysis, M.W.; Investigation, M.M.; Resources, A.W.; Data Curation, J.S., T.W., M.M.; Writing-Original Draft Preparation, J.S., T.W.; Writing - Review \& Editing, M.W., H.M.; Visualization, M.W.; Supervision, G.M.; Project Administration, H.M.; Funding Acquisition, M.W., G.M.

Funding: Case reports are a part of the research project titled "Evaluation of the Quality of Sleep, Endothelial Function, Cardiovascular Risk, Thyroid Function, a Function of Masticatory Muscles and Psycho-emotional State of Patients with Sleep Bruxism". The project was performed in the Department and Clinic of Internal and Occupational Diseases and Hypertension, Wroclaw Medical University, Wroclaw, Poland. The project was co-financed from funds for young researchers obtained from the Wroclaw Medical University (STM.B022.17.011). The project was accepted by the local Ethical Committee (ID KB-195/2017) and registered on the ClinicalTrials.gov (ID WMU1/2017).

Acknowledgments: Carlos H. Schenck critically reviewed the manuscript before submission.

Conflicts of Interest: The authors declare no conflict of interest.

\section{References}

1. Fleetham, J.A.; Fleming, J.A. Parasomnias. CMAJ 2014, 186, E273-E280. [CrossRef] [PubMed]

2. American Academy of Sleep Medicine. International Classification of Sleep Disorders, 3rd ed.; American Academy of Sleep Medicine: Darien, IL, USA, 2014.

3. American Psychiatric Association. Diagnostic and Statistical Manual of Mental Disorders. In DSM-5, 5th ed.; American Psychiatric Association: Arlington, VA, USA, 2013.

4. Zucconi, M.; Ferri, R. Classification of Sleep Disorders. In Sleep Medicine Textbook; European Sleep Research Society: Regensburg, Germany, 2014; pp. 95-109.

5. Muza, R.; Lawrence, M.; Drakatos, P. The reality of sexsomnia. Curr. Opin. Pulm. Med. 2016, 22, 576-582. [CrossRef] [PubMed]

6. Organ, A.; Fedoroff, J.P. Sexsomnia: Sleep sex research and its legal implications. Curr. Psychiatry Rep. 2015, 17, 34. [CrossRef] [PubMed]

7. Andersen, M.L.; Poyares, D.; Alves, R.S.; Skomro, R.; Tufik, S. Sexsomnia: Abnormal sexual behavior during sleep. Brain Res. Rev. 2007, 56, 271-282. [CrossRef] [PubMed]

8. Ebrahim, I.O. Somnambulistic sexual behaviour (sexsomnia). J. Clin. Forensic Med. 2006, 13, 219-224. [CrossRef] [PubMed] 
9. Schenck, C.H.; Arnulf, I.; Mahowald, M.W. Sleep and sex: What can go wrong? A review of the literature on sleep related disorders and abnormal sexual behaviors and experiences. Sleep 2007, 30, 683-702. [CrossRef] [PubMed]

10. Gomis Devesa, A.J.; Ortega Albás, J.J.; Denisa Ghinea, A.; Carratalá Monfort, S. Sexsomnia and sleep eating secondary to sodium oxybate consumption. Neurologia 2016, 31, 355-356. [CrossRef] [PubMed]

11. Ariño, H.; Iranzo, A.; Gaig, C.; Santamaria, J. Sexsomnia: Parasomnia associated with sexual behaviour during sleep. Neurologia 2014, 29, 146-152. [CrossRef] [PubMed]

12. Béjot, Y.; Juenet, N.; Garrouty, R.; Maltaverne, D.; Nicolleau, L.; Giroud, M.; Didi-Roy, R. Sexsomnia: An uncommon variety of parasomnia. Clin. Neurol. Neurosurg. 2010, 112, 72-75. [CrossRef] [PubMed]

13. Krol, D.G. Sexsomnia during treatment with a selectiveserotonin reuptake inhibitor. Tijdschr. Psychiatr. (J. Psychiatry) 2008, 50, 735-739.

14. Meira, E.; Cruz, M.; Soca, R. Sexsomnia and REM-Predominant obstructive sleep apnea effectively treated with a mandibular advancement device. Sleep Sci. 2016, 9, 140-141. [CrossRef] [PubMed]

15. Khawaja, I.S.; Hurwitz, T.D.; Schenck, C.H. Sleep-related abnormal sexual behavior (sexsomnia) successfully treated with a mandibular advancement device: A case report. J. Clin. Sleep Med. 2017, 13, 627-628. [CrossRef] [PubMed]

16. Schenck, C.H.; Mahowald, M.W. Parasomnias Associated with sleep-disordered breathing and its therapy, including sexsomnia as a recently recognized parasomnia. Somnologie 2008, 12, 38-49. [CrossRef]

17. Della Marca, G.; Dittoni, S.; Frusciante, R.; Colicchio, S.; Losurdo, A.; Testani, E.; Buccarella, C.; Modoni, A.; Mazza, S.; Mennuni, G.F.; et al. Abnormal sexual behavior during sleep. J. Sex. Med. 2009, 6, 3490-3495. [CrossRef] [PubMed]

18. Inoue, Y. Sleep-related eating disorder and its associated conditions. Psychiatry Clin. Neurosci. 2015, 69, 309-320. [CrossRef] [PubMed]

19. Yeh, S.B.; Schenck, C.H. Sexsomnia: A case of sleep masturbation documented by video-polysomnography in a young adult male with sleepwalking. Sleep Sci. 2016, 9, 65-68. [CrossRef] [PubMed]

20. Soca, R.; Keenan, J.C.; Schenck, C.H. Parasomnia overlap disorder with sexual behaviors during sleep in a patient with obstructive sleep apnea. J. Clin. Sleep Med. 2016, 12, 1189-1191. [CrossRef] [PubMed]

21. Pelin, Z.; Yazla, E. Abnormal sexual behavior during sleep in temporal lobe epilepsy: A case report. Balkan Med. J. 2012, 29, 211-213. [CrossRef] [PubMed]

22. Cicolin, A.; Tribolo, A.; Giordano, A.; Chiarot, E.; Peila, E.; Terreni, A.; Bucca, C.; Mutani, R. Sexual behaviors during sleep associated with polysomnographically confirmed parasomnia overlap disorder. Sleep Med. 2011, 12, 523-528. [CrossRef] [PubMed]

23. Shapiro, C.M.; Trajanovic, N.N.; Fedoroff, J.P. Sexsomnia-A new parasomnia? Can. J. Psychiatry 2003, 48, 311-317. [CrossRef] [PubMed]

24. Irfan, M.; Schenck, C.H. Sleep-related orgasms in a 57-year-old woman: A case report. J. Clin. Sleep Med. 2018, 14, 141-144. [CrossRef] [PubMed]

25. Mioč, M.; Antelmi, E.; Filardi, M.; Pizza, F.; Ingravallo, F.; Nobili, L.; Tassinari, C.A.; Schenck, C.H.; Plazzi, G. Sexsomnia: A diagnostic challenge, a case report. Sleep Med. 2018, 43, 1-3. [CrossRef] [PubMed]

26. Lobbezoo, F.; Ahlberg, J.; Glaros, A.G.; Kato, T.; Koyano, K.; Lavigne, G.J.; de Leeuw, R.; Manfredini, D.; Svensson, P.; Winocur, E. Bruxism defined and graded: An international consensus. J. Oral Rehabil. 2013, 40, 2-4. [CrossRef] [PubMed]

27. Manfredini, D.; Winocur, E.; Guarda-Nardini, L.; Paesani, D.; Lobbezoo, F. Epidemiology of bruxism in adults: A systematic review of the literature. J. Orofac. Pain 2013, 27, 99-110. [CrossRef] [PubMed]

28. Manfredini, D.; Serra-Negra, J.; Carboncini, F.; Lobbezoo, F. Current Concepts of Bruxism. Int. J. Prosthodont. 2017, 30, 437-438. [CrossRef] [PubMed]

29. Ovcharov, V.K. International Classification of Diseases, Tenth Revision, Clinical Modification (ICD-10-CM). Available online: http://www.cdc.gov/nchs/icd/icd9cm.htm (accessed on 3 August 2018).

30. Lobbezoo, F.; Ahlberg, J.; Raphael, K.G.; Wetselaar, P.; Glaros, A.G.; Kato, T.; Santiago, V.; Winocur, E.; De Laat, A.; De Leeuw, R.; et al. International consensus on the assessment of bruxism: Report of a work in progress. J. Oral Rehabil. 2018. [CrossRef] [PubMed] 
31. Schiffman, E.; Ohrbach, R.; Truelove, E.; Look, J.; Anderson, G.; Goulet, J.P.; List, T.; Svensson, P.; Gonzalez, Y.; Lobbezoo, F; ; et al. Diagnostic Criteria for Temporomandibular Disorders (DC/TMD) for clinical and research applications: Recommendations of the International RDC/TMD Consortium Network and Orofacial Pain Special Interest Group. J. Oral Facial Pain Headache 2014, 28, 6-27. [CrossRef] [PubMed]

32. Berry, R.B.; Budhiraja, R.; Gottlieb, D.J.; Gozal, D.; Iber, C.; Kapur, V.K.; Marcus, C.L.; Mehra, R.; Parthasarathy, S.; Quan, S.F.; et al. Rules for scoring respiratory events in sleep: Update of the 2007 AASM Manual for the scoring of sleep and associated events. J. Clin. Sleep Med. 2012, 8, 597-619. [CrossRef] [PubMed]

(C) 2018 by the authors. Licensee MDPI, Basel, Switzerland. This article is an open access article distributed under the terms and conditions of the Creative Commons Attribution (CC BY) license (http://creativecommons.org/licenses/by/4.0/). 